\title{
Declínio do estado nutricional infanto-juvenil durante o tratamento oncológico: seus agravantes e a relevância da terapia nutricional
}

\author{
Decline of the nutritional status of children and youth during cancer treatment: its aggravations \\ and the relevance of nutritional therapy
}

Deterioro del estado nutricional de los niños durante el tratamiento del cáncer: sus agravamientos y la relevancia de la terapia nutricional

Recebido: 01/12/2021 | Revisado: 07/12/2021 | Aceito: 08/12/2021 | Publicado: 16/12/2021

\author{
Natália Pereira Ferreira \\ ORCID: https://orcid.org/0000-0003-0423-2311 \\ Centro Universitário de Brasília, Brasil \\ E-mail: natii.npf@gmail.com \\ Jamila de Almada Pardo \\ ORCID: https://orcid.org/0000-0002-0178-2177 \\ Centro Universitário de Brasília, Brasil \\ E-mail: jamilapardo@gmail.com \\ Ana Lúcia Ribeiro Salomon \\ ORCID: https://orcid.org/0000-0002-1181-5948 \\ Centro Universitário de Brasília, Brasil \\ E-mail: ana.salomon@gmail.com
}

\begin{abstract}
Resumo
A desnutrição tem sido algo muito comum em crianças e adolescentes submetidos a tratamentos oncológicos. Trata-se de uma doença causada pela falta de nutrientes, gerando um déficit e ocasionando uma série de danos ao organismo, podendo até levar à morte caso não tratada. Devido aos seus efeitos adversos, a nutrição, com a terapia nutricional, possui um papel importantíssimo na manutenção e melhora do estado nutricional desses pacientes. O presente estudo teve como objetivo principal alertar a respeito da incidência da desnutrição em pacientes oncológicos e da importância da terapia nutricional nesse momento. Tratou-se de um estudo observacional, do tipo transversal descritivo. A coleta de dados foi feita por meio da aplicação de um questionário online com pais ou responsáveis de crianças e/ou adolescentes na faixa etária de 7 a 19 anos, onde foram obtidas informações como dados antropométricos, tipo de tratamento em realização e os sintomas apresentados. Essas informações resultaram em uma prevalência de neoplasias no público masculino, com média de idade de 12,5 anos, pacientes, em sua maioria, de quimioterapia e radioterapia. Houve perda de peso considerável, em 92,9\% dos participantes da pesquisa, devido aos diversos sintomas gastrointestinais e orais apresentados e com isso, percebeu-se a importância da terapia nutricional nesse tratamento, com o intuito de minimizar o declínio no estado nutricional do paciente.
\end{abstract}

Palavras-chave: Desnutrição; Câncer; Tratamento; Nutrição; Terapia nutricional.

\begin{abstract}
Malnutrition has been very common in children and adolescents undergoing cancer treatments. It is a disease caused by a lack of nutrients, generating a deficit and causing a series of damages to the body, which can even lead to death if left untreated. Due to its adverse effects, nutrition, with nutritional therapy, has a very important role in maintaining and improving the nutritional status of these patients. The main objective of this study was to raise awareness about the incidence of malnutrition in cancer patients and the importance of nutritional therapy at that time. This was an observational, cross-sectional descriptive study. Data collection was carried out through the application of an online questionnaire with parents or guardians of children and/or adolescents aged 7 to 19 years, where information was obtained such as anthropometric data, type of treatment in progress and the symptoms presented. This information resulted in a prevalence of neoplasms in the male population, with a mean age of 12.5 years, patients, mostly undergoing chemotherapy and radiotherapy. There was considerable weight loss, in $92.9 \%$ of the research participants, due to the various gastrointestinal and oral symptoms presented and with this, the importance of nutritional therapy in this treatment was realized, in order to minimize the decline in the nutritional status of the patient.
\end{abstract}

Keywords: Malnutrition; Cancer; Treatment; Nutrition; Nutritional therapy. 


\begin{abstract}
Resumen
La desnutrición ha sido muy común en niños y adolescentes sometidos a tratamientos contra el cáncer. Es una enfermedad provocada por la falta de nutrientes, que genera un déficit y provoca una serie de daños en el organismo, que incluso pueden llegar a la muerte si no se tratan. Por sus efectos adversos, la nutrición, junto con la terapia nutricional, tiene un papel muy importante en el mantenimiento y mejora del estado nutricional de estos pacientes. El principal objetivo de este estudio fue concienciar sobre la incidencia de desnutrición en pacientes con cáncer y la importancia de la terapia nutricional en ese momento. Se trata de un estudio descriptivo transversal, observacional. La recogida de datos se realizó mediante la aplicación de un cuestionario online con padres o tutores de niños y / o adolescentes de 7 a 19 años, donde se obtuvo información como datos antropométricos, tipo de tratamiento en curso y los síntomas presentados. Esta información resultó en una prevalencia de neoplasias en la población masculina, con una edad media de 12,5 años, pacientes, en su mayoría sometidos a quimioterapia y radioterapia. Hubo una pérdida de peso considerable, en el $92,9 \%$ de los participantes de la investigación, debido a los diversos síntomas gastrointestinales y orales presentados y con ello, se tomó conciencia de la importancia de la terapia nutricional en este tratamiento, con el fin de minimizar el deterioro del estado nutricional de los pacientes.
\end{abstract}

Palabras clave: Desnutrición; Cáncer; Tratamiento; Nutrición; Terapia nutricional.

\title{
1. Introdução
}

Câncer é uma palavra utilizada para se referir a mais de cem tipos de patologias malignas, porém todas possuem algo que se assemelham. Nele há a ocorrência de um crescimento celular descontrolado fazendo com que essas células geralmente sejam bastante agressivas e ocorra a formação de tumores, podendo, estes, serem isolados ou se alastrar para outros órgãos e tecidos, fenômeno denominado de metástase (INCA, 2020).

O câncer infanto-juvenil trata-se de um problema global de saúde, pois não se limita apenas a países subdesenvolvidos ou em desenvolvimento, englobando países desenvolvidos também. Sua incidência varia de 50 a 200 casos por milhão no mundo e no Brasil é de 154,3 pessoas afetadas por milhão (Feliciano, Santos \& Pombo-de-Oliveira, 2019).

A desnutrição trata-se de uma doença multifatorial na qual há um déficit de nutrientes no organismo, podendo gerar danos a diversos órgãos do paciente e, consequentemente, até a morte, caso não seja tratada (Carvalho et al., 2005).

Em pacientes passando pelo tratamento oncológico, a incidência dos quadros de desnutrição impacta tanto reduzindo sua qualidade de vida e resposta ao tratamento, quanto elevando o risco de ocorrer intoxicação pela quimioterapia, resultando na redução da possibilidade de sobrevivência do indivíduo (Smiderle \& Gallon, 2012).

O declínio do estado nutricional da pessoa depende estatisticamente das características do câncer, podendo variar a prevalência da desnutrição entre $6 \%$ em infantes com doenças localizadas, a $50 \%$ em tumores sólidos em estágios avançados (Silva Mag et al., 2018).

Em crianças e adolescentes, portadores de neoplasias malignas, os principais motivos do desenvolvimento da desnutrição estão relacionados à redução do consumo alimentar, variações no gasto energético, alterações na absorção e metabolização de nutrientes pela ordem fisiológica em que ocorrem, sendo capaz de resultar em complicações como infecções, toxicidade oral, problemas gastrointestinais e nefrotoxicidade relacionado ao tratamento oncológico (Garófolo et al., 2005).

O público infanto-juvenil possui uma grande variação em sua necessidade energética gerada por cada faixa etária e exatamente por isso a terapia nutricional individualizada é tão importante. O propósito terapêutico tem como alvo, garantir quantidades apropriadas de nutrientes e fluidos para manutenção das funções vitais, obtenção de energia e a homeostase (Garófolo, 2005).

O diagnóstico de câncer é destacado por sua agressividade trazendo várias complicações, como a desnutrição (Souza \& Fortes, 2012). Diante disso percebe-se a importância da terapia nutricional para minimizar os sintomas gerados em decorrência do tratamento oncológico.

Diante do exposto, este estudo teve por objetivo alertar sobre a incidência da desnutrição nos pacientes oncológicos e mostrar a importância da intervenção nutricional nesse momento. 


\section{Metodologia}

Foi realizado um estudo do tipo observacional transversal descritivo.

Neste estudo participaram 14 crianças e/ou adolescentes com idade de 7 a 19 anos.

O estudo foi realizado por meio da aplicação de um questionário online com pais ou responsáveis de crianças e/ou adolescentes na faixa etária de 7 a 19 anos, frequentadores de clínica oncológica para a realização do seu tratamento.

Para participação no estudo o paciente devia ser portador de câncer, possuir faixa etária descrita acima, estar realizando algum tipo de tratamento oncológico e apresentar um declínio do estado nutricional. Após a pesquisa, foi divulgado, nas redes sociais, um e-book sobre alimentos funcionais e receitas que auxiliam a terapia nutricional deste público.

Foi aplicado o questionário contendo as seguintes informações: dados pessoais (idade, sexo), dados antropométricos (peso usual e atual, altura), sintomas do paciente (náuseas, vômitos, diarreia, hiporexia, constipação, estomatite, herpes bucal, mucosite, xerostomia, odinofagia, cistite, dermatites, alopecia, azia, gases, rouquidão, problemas dentários, trismo, dispneia), tipo de tratamento utilizado (quimioterapia, radioterapia, imunoterapia, iodoterapia), assim como tipos de quimioterapia (adjuvante, neoadjuvante, paliativa, curativa, prévia), radioterapia (externa ou convencional e interna ou braquiterapia) e imunoterapia (Anticorpos monoclonais, Inibidores dos check-points imunes, Imunoterapias não específicas), a terapia nutricional utilizada (oral, enteral, parenteral e suplementação) e tipo de dieta (geral, líquida, pastosa, hiperproteica, hipercalórica, hipossódica). Nas questões referentes aos sintomas, tipos de tratamento, terapia nutricional utilizada e tipo de dieta, era possível marcar mais de uma opção.

O período da coleta de dados foi de 27 de Setembro a 21 de Outubro de 2021, totalizando vinte e cinco dias.

Os dados foram analisados com o auxílio das ferramentas do Google: Planilhas e Formulários, mediante as quais os dados foram apresentados em forma de tabelas e gráficos. As classificações do IMC e do escore $\mathrm{Z}$ foram feitas com base no Software WHO Anthro Plus, que trouxe toda a análise de estado nutricional.

Para participar da pesquisa o participante deveria ser responsável por uma criança ou adolescente portador de câncer, que possuísse de 7 a 19 anos de idade, e que estivesse fazendo algum tipo de tratamento oncológico.

Foram excluídos do estudo, formulários com dados incompletos, que inviabilizaram a obtenção das variáveis especificadas. Também foram excluídos aqueles participantes que, durante o processo, decidiram desistir da participação no estudo.

Os procedimentos metodológicos do presente trabalho foram preparados dentro dos procedimentos éticos e científicos fundamentais, como disposto na Resolução N. ${ }^{\circ}$ 466, de 12 de dezembro de 2012 do Conselho Nacional de Saúde do Ministério da Saúde, sendo aprovados pelo Comitê de Ética em Pesquisa do UniCEUB conforme protocolo no 4.987.643/21.

A coleta de dados foi iniciada apenas após a aprovação do referido comitê e ciência da concordância de participação com o TCLE contido no formulário online. Na execução e divulgação dos resultados foi garantido o total sigilo da identidade dos participantes e a não discriminação ou estigmatização dos sujeitos da pesquisa, além da conscientização dos sujeitos quanto à publicação de seus dados. A todos os participantes foi solicitado o preenchimento do aceite digital do termo de consentimento livre e esclarecido (TCLE) e somente após este aceite, os sujeitos da pesquisa conseguiram ter acesso ao questionário.

\section{Resultados}

Foram colhidas através do questionário online, aplicado por meio do google forms, 18 respostas, sendo 4 eliminadas por não se enquadrarem na faixa etária determinada. Entre as 14 respostas analisadas $57,1 \%$ eram de participantes do sexo masculino e 42,9\% do sexo feminino, sendo a média etária de 12,5 anos com desvio padrão de 3,13. 
Em relação aos dados antropométricos, a média de peso atual, durante o tratamento oncológico, esteve em 47 quilos, diferente da média do peso usual ficando em 53 quilos. No desvio padrão atual obteve-se 14,89, não muito diferente do usual que ficou em torno de 14,53. No quesito altura dos participantes, a média apresentou-se em 1,60 metros, com o desvio padrão de 19,87 .

Na classificação do estado nutricional pelo software WhoAnthro Plus, 4 pacientes $(28,6 \%)$ se enquadraram em baixo peso, com igual distribuição entre os sexos, $6(42,8 \%)$ em eutrofia, com igual distribuição entre os sexos, e 4 (28,6\%) em sobrepeso (sendo 1 do sexo feminino e 3 do sexo masculino). Entretanto, ao se analisar a perda de peso apresentada pelos participantes, grande marcador para a identificação de risco para a desnutrição, 100\% dos indivíduos a apresentaram, conforme demonstrado na Tabela 1, sendo que 13 pacientes $(92,9 \%)$ tiveram perdas superiores a 5\%, um parâmetro já considerado para a definição da caquexia do câncer.

Tabela 1 - Relação: Percentual de perda de peso (PP\%) x Sexo. Brasília - DF, 2021.

\begin{tabular}{ccc}
\hline PP\% & Feminino & Masculino \\
\hline $\mathbf{0}-\mathbf{5}$ & 0 & 1 \\
$\mathbf{5}-\mathbf{1 0}$ & 1 & 0 \\
$\mathbf{1 0}-\mathbf{1 5}$ & 0 & 2 \\
mais de 15 & 5 & 5 \\
& $\mathbf{6}$ & $\mathbf{8}$ \\
\hline
\end{tabular}

Fonte: Autores (2021).

Nos tratamentos realizados, vale ressaltar que, em virtude da possibilidade de associação de terapias para melhor manejo da doença, mais de uma opção de resposta era possível. A terapia antineoplásica mais utilizada neste estudo foi a quimioterapia, por 13 participantes (92,9\%), sendo que 12 deles $(85,7 \%)$ do tipo curativa, $1(7,1 \%)$ também fez uso da prévia e $1(7,1 \%)$ adjuvante. A segunda terapia mais utilizada foi a radioterapia, por 6 pessoas $(42,9 \%)$, sendo que todos eles fazem uso do tipo externa ou, também, conhecida como convencional. As demais terapias empregadas podem ser observadas na Figura 1.

Figura 1 - Tratamentos Utilizados. Brasília - DF, 2021.

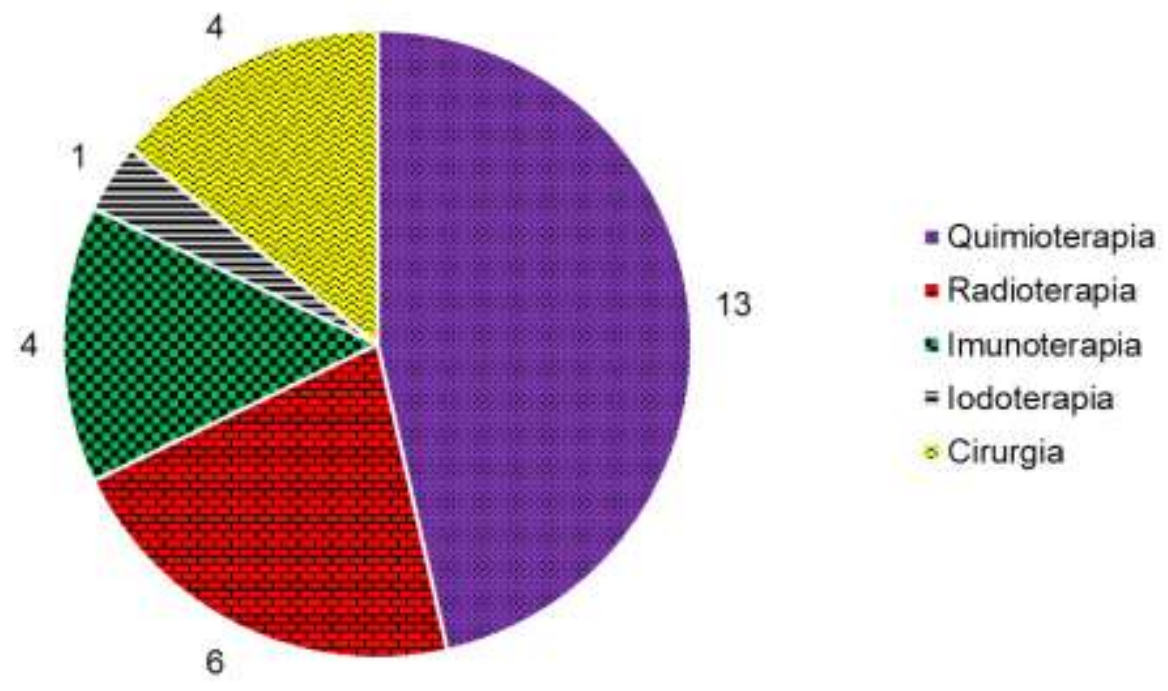


Fonte: Autores (2021).

Os sintomas do trato gastro intestinal mais predominantes foram náuseas (100\%), seguido de azia (78,6\%), flatulências $(64,3 \%)$ e outros (Figura 2). Também foram relatados nos sintomas de alterações orais, a mucosite (71,4\%), a xerostomia $(50 \%)$ e outros (Figura 3$)$.

Figura 2 - Sintomas Gastrointestinais. Brasília - DF, 2021.

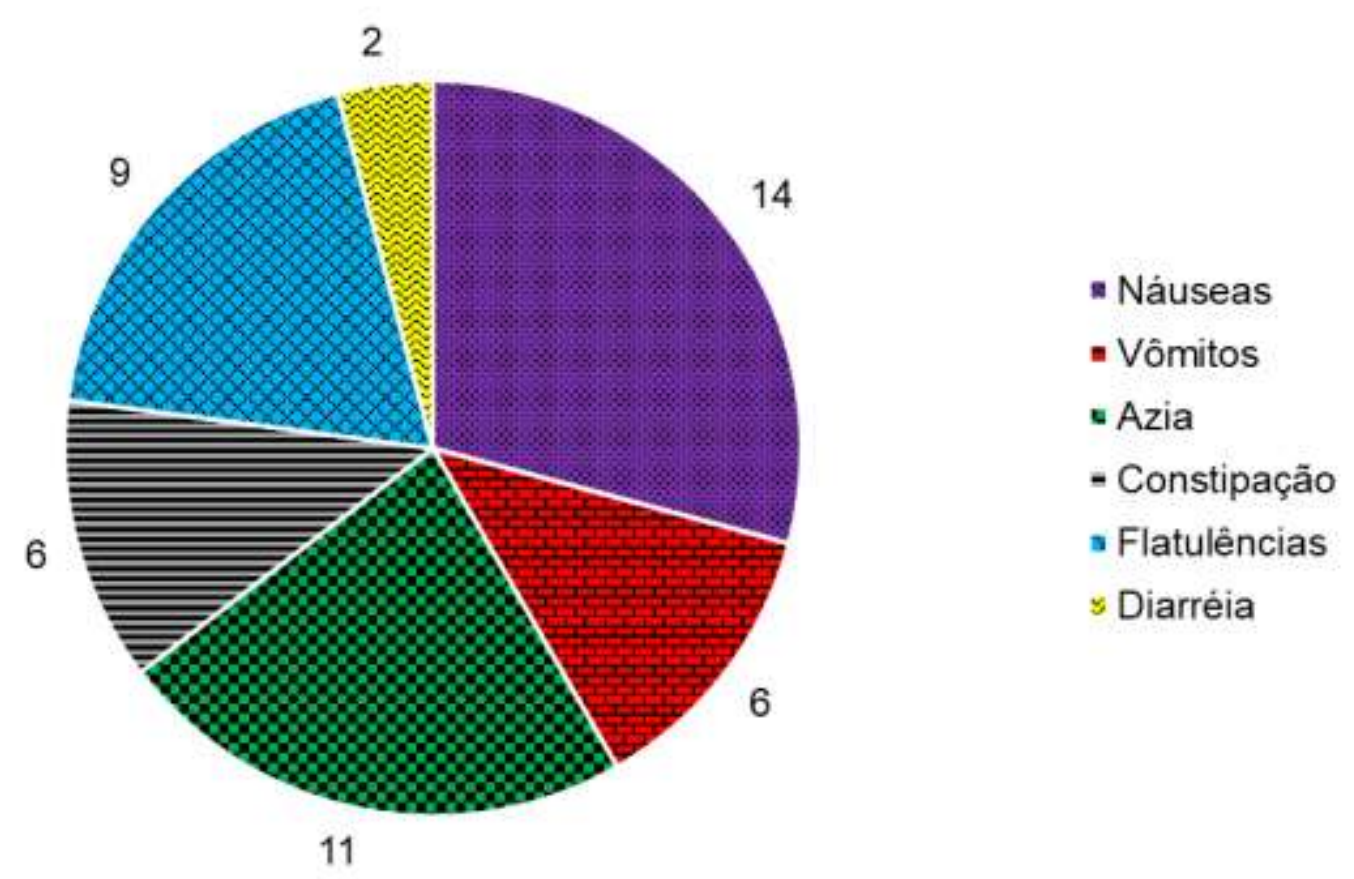

Fonte: Autores (2021).

Figura 3 - Sintomas Orais. Brasília - DF, 2021.

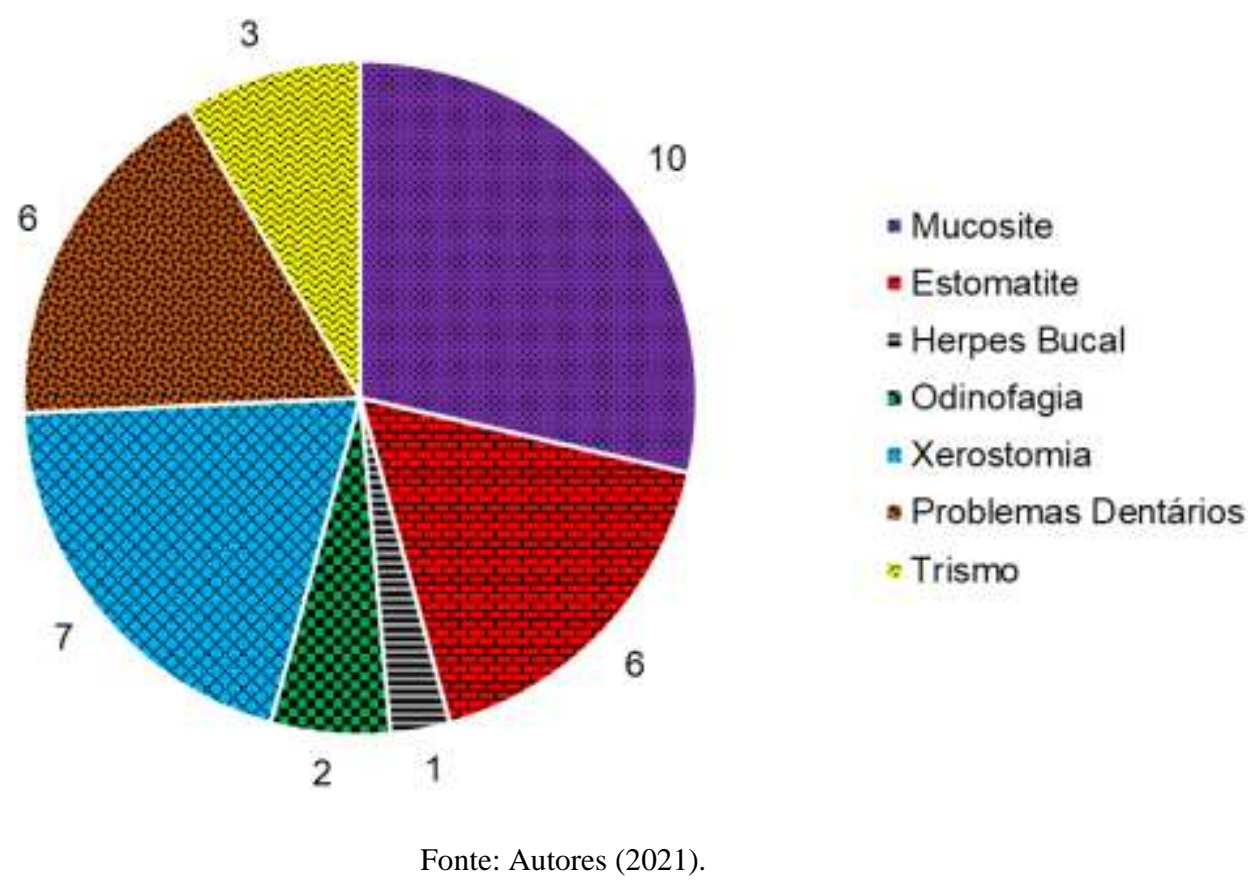


Dentre sintomas diversos os mais recorrentes foram fadiga (100\%), alopecia $(85,7 \%)$, cefaleia $(85,7 \%)$, hiporexia $(78,6 \%)$, dermatites $(64,3 \%)$, rouquidão $(42,9 \%)$, febre $(35,7 \%)$, trismo $(21,4 \%)$ e dispneia $(7,1 \%)$.

Na Tabela 2 é apresentada a relação entre os tipos de tratamentos empregados e os sintomas relatados pelos seus usuários.

Tabela 2 - Relação: Tratamentos x Sintomas. Brasília - DF, 2021.

\begin{tabular}{lcccc}
\hline & Quimioterapia & Radioterapia & Imunoterapia & Iodoterapia \\
\hline Náuseas & 13 & 6 & 4 & 1 \\
Vômitos & 6 & 4 & 1 & 0 \\
Constipação & 5 & 2 & 0 & 1 \\
Azia & 11 & 5 & 3 & 0 \\
Flatulências & 9 & 5 & 3 & 0 \\
Estomatite & 6 & 2 & 3 & 0 \\
Mucosite & 10 & 4 & 3 & 0 \\
Xerostomia & 6 & 3 & 2 & 0 \\
Problemas dentários & 6 & 4 & 3 & 0 \\
Rouquidão & 6 & 3 & 3 & 0 \\
Alopecia & 12 & 6 & 4 & 0 \\
Dermatites & 9 & 5 & 3 & 0 \\
Hiporexia & 11 & 6 & 4 & 1 \\
Fadiga & 13 & 6 & 4 & 1 \\
Cefaleia & 11 & 6 & 4 & $\mathbf{4}$ \\
& & & $\mathbf{4 4}$ & \\
\hline
\end{tabular}

Fonte: Autores (2021).

Sobre a terapia nutricional utilizada, 10 pessoas $(83,33 \%)$ informaram ser via oral, dentro dessas 5 pessoas realizaram suplementação $(50 \%)$, a via enteral era utilizada por 2 pacientes (16,67\%). Para gerar o resultado a respeito da terapia nutricional utilizada, foram consideradas somente respostas de 12 participantes, devido ao fato de 2 deles terem respondido de forma incoerente.

\section{Discussão}

Pacientes com câncer, em sua maioria, estão sob o risco de desenvolver desnutrição, agravando sua condição clínica em qualquer fase do tratamento. A desnutrição é uma condição inadequada do estado nutricional, e associada ao tratamento possui efeitos adversos que podem agravar o estado do paciente, especialmente em crianças e adolescentes (Gomes, 2016).

Neste estudo a presença de câncer com maior frequência ocorreu no sexo masculino $(57,1 \%)$ com média etária de 12,5 \pm 3,13 anos, resultado parecido foi encontrado no estudo realizado em 30 pacientes internados no instituto de Medicina Integral Prof. Fernando Figueira em Recife-PE, onde observou em sua maioria, cerca de $60 \%$ dos pacientes, como sendo do sexo masculino, na média etária de 7,1 \pm 4,3 anos (Pereira et al., 2017). 
No mesmo sentido há a pesquisa realizada no Hospital do câncer no município de São Luís - MA, onde foram avaliadas 53 crianças, sendo $81,1 \%$ meninos, destes $52,8 \%$ estavam na faixa etária de 7 a 8 anos. Em contrapartida, na pesquisa realizada para investigar a prevalência de desnutrição em 42 crianças de 0 a 12 anos de idade, no Município de Jundiaí, constatou-se que 57,1\% eram do gênero feminino (Caram et al., 2014).

$\mathrm{Na}$ avaliação realizada de IMC por idade, Sousa e colaboradores (2016) constataram um total de $66 \%$ de crianças eutróficas, sendo que 92,5\% possuíam estatura adequada para a idade, apenas apresentando risco nutricional para 23,6\% dos meninos e $20 \%$ das meninas. Por outro lado, neste estudo observou-se que $42,86 \%$ dos entrevistados possuía a estatura adequada para a idade sendo a altura elevada representando $28,57 \%$, a baixa $14,28 \%$ e a estatura de risco $14,28 \%$. Já no estudo de Pereira e colaboradores (2017), o estado nutricional apontou que houveram déficit nos seguintes indicadores: 4,2\% peso/idade, $3,3 \%$ altura/idade e $16,7 \%$ índice de massa corporal/idade.

Araújo e colaboradores (2012) também realizaram um estudo utilizando o método transversal com 30 pacientes oncológicos menores de 18 anos, que estavam realizando quimioterapia, onde avaliaram o estado nutricional, obtendo o resultado de $10 \%$ apresentando baixa estatura, segundo os indicadores de altura/idade.

Já no estudo de Carraro et al. (2012) foram acompanhadas 19 crianças e adolescentes no Hospital de Clínicas de Porto Alegre, na faixa etária entre 4 e 18 anos. Esses pacientes foram classificados de acordo com os valores de escore-z no tocante ao índice de massa corporal para idade, onde apresentaram 10,5\% com magreza, 52,6\% possuíam eutrofia e 36,8\% apresentando excesso de peso. Já nesta pesquisa o escore-z ficou dividido em 28,57\% com magreza, 42,86\% em eutrofia e $28,57 \%$ possuindo excesso de peso.

Diferente deste estudo os autores Lemos e colaboradores (2014) avaliaram 1.154 pacientes no Instituto de Oncologia Pediátrica da Universidade Federal de São Paulo, com a faixa de idade entre 10 e 24 anos, onde, em sua maioria, eram do sexo masculino com 53,09\%, os dados antropométricos revelaram que 10,85\% apresentavam IMC por idade abaixo do adequado. No entanto, os autores não apresentaram dados sobre os tipos de tratamentos aplicados, sintomas ou capacidade de ingestão oral.

Vale ressaltar que os parâmetros antropométricos para a avaliação do estado nutricional de crianças e adolescentes, recomendados pela Organização Mundial de Saúde traduzem a situação atual da condição antropométrica. Por outro lado, ao analisarmos parâmetros mais sensíveis para a identificação do risco para desenvolvimento de desnutrição, como a perda de peso, verificamos que todos os pacientes deste estudo apresentaram essa perda, sendo que 92,9\% perderam mais de $5 \%$ em relação ao seu peso usual, o que, somado a alterações na capacidade de assimilação de nutrientes (por sintomas gastrointestinais) e a condição inflamatória da doença, pode apontar para processos de caquexia oncológica.

Em comparação ao estudo realizado por Sgarbieri et al. (2006), com 45 crianças diagnosticadas com leucemia, 5 crianças apresentaram uma perda ponderal de $11,1 \%$ estando na faixa de desnutridos e 6,7\% dos pacientes apresentaram um escore de peso/estatura inferior a -2, dados informados durante a manutenção da quimioterapia, nesses pacientes.

No estudo realizado por Mutti et al. (2008) de análise de 160 prontuários de crianças e adolescentes, foi encontrado a predominância no sexo masculino $(94,64 \%)$ e em relação aos tratamentos realizados, identificou-se que $45 \%$ utilizaram unicamente da quimioterapia, $38 \%$ estiveram associadas a outras terapias, como, a radioterapia $(25,17 \%)$ e cirurgia $(22,15 \%)$. Em comparação a esse estudo obteve-se a utilização da quimioterapia em 92,9\% dos participantes, sendo que em 71,43\% esteve associada a algum outro tratamento antineoplásico, tais como, 42,86\% à radioterapia e 21,43\% à cirurgia.

Avaliando a referência de efeitos colaterais das terapias antineoplásicas apresentadas pela amostra deste estudo, com potencial para impactar negativamente na assimilação de nutrientes contribuindo para a desnutrição, os sintomas se dividiram em náuseas em $100 \%$, hiporexia em $78,6 \%$, mucosite em $71,4 \%$, a xerostomia em $50 \%$, vômitos em $42,9 \%$, estomatite em $42,9 \%$, constipação intestinal em $42,9 \%$, febre em $35,7 \%$, diarreia em $14,3 \%$ e herpes bucal em $7,1 \%$. Tratam-se de queixas 
importantes que contribuem para uma menor ingestão alimentar que, em contraposição ao gasto energético elevado, reforça o risco para a caquexia. Tal sintomatologia fez com que apenas $35,7 \%$ dos pacientes apresentassem uma normalidade na sua alimentação.

Em uma pesquisa realizada em Jundiaí, 57,1\% eram do gênero feminino, onde 52,4\% destas apresentaram desnutrição. A alimentação normalizada ficou em $47,8 \%$, os sintomas mais frequentes foram, vômitos com $71,4 \%$, estomatite em 47,65\%, diarreia em 45,2\%, náuseas em 42,9\%, herpes bucal em 14,3\% e constipação intestinal em 9,5\% (Caram et al., 2014)

No quesito de terapia nutricional utilizada pelos pacientes deste estudo, das 12 respostas obtidas, 41,7\% utilizavam a suplementação oral e 16,7\% estavam em nutrição enteral. Vale frisar que, embora houvesse emprego de terapia nutricional em $58,4 \%$ da amostra, todos apresentaram perda de peso, o que chama atenção para uma provável introdução tardia da terapia. Outro ponto importante é que, enquanto $35,7 \%$ dos pacientes referiram a manutenção de uma alimentação normal, $41,6 \%$ estavam sem terapia nutricional especializada, o que resulta em 5,9\% com inadequação terapêutica.

Faz-se fundamental o estabelecimento de um diagnóstico nutricional precoce, baseado não somente em parâmetros antropométricos (tradutores da situação atual), mas também aliado a métodos subjetivos de avaliação nutricional, contemplando fatores que venham a colocar o paciente em risco para a desnutrição, incluindo a perda de peso (relatada por $100 \%$ da amostra), sintomas que impactem a ingestão e/ou o aproveitamento de nutrientes, estresse metabólico da doença e outras comorbidades e avaliação de composição corporal, sobretudo no que tange à massa magra. Sem esses parâmetros, incorre-se em risco de deterioração nutricional dos pacientes, promovendo o desenvolvimento da desnutrição ou mesmo de quadros de caquexia, onde, por sua vez, impactam negativamente a resposta aos tratamentos utilizados (promovendo menor tolerância por aumento de toxicidade das terapias), na qualidade de vida e na própria sobrevida desta população.

A identificação precoce do diagnóstico nutricional permite que a terapia nutricional seja implementada em tempo adequado e, preferencialmente, antes do início dos tratamentos antineoplásicos, para que se obtenha melhor resposta dos mesmos, bem como se alcance um prognóstico mais favorável.

\section{Conclusão}

No presente estudo, foi analisado o declínio do estado nutricional de crianças e adolescentes, de 7 a 19 anos, em tratamento oncológico. Ele teve o intuito de avaliar a incidência de desnutrição nesse público e mostrar a importância da terapia nutricional no auxílio do tratamento.

Os resultados nele encontrados foram a maior prevalência de câncer no público masculino (57,1\%), em uma média etária de 12,5 anos (com desvio padrão de 3,13), os tratamentos mais utilizados foram a quimioterapia e a radioterapia, os quais geraram muitos sintomas sendo gastrointestinais, orais, entre outros. Os sintomas mais recorrentes foram náuseas, azia, flatulências, mucosite, xerostomia, cefaleia e fadiga.

Tais resultados mostram dados alarmantes, tanto de forma numérica quanto de forma interpretativa. É possível perceber que a importância da terapia nutricional pode estar sendo subestimada, tendo seu início tardiamente. Esse fato se mostrou real quando analisou-se o percentual de perda de peso, onde cerca de 92,9\% dos participantes tiveram uma perda maior que 5\%. Isso, associado com vários sintomas que influenciam na ingestão alimentar, já evidencia um quadro de sarcopenia e/ou caquexia causada pelo tratamento antineoplásico.

Portanto, analisando os dados gerados nesse estudo, observou-se a importância da intervenção nutricional precoce e contínua, no tratamento antineoplásico, a fim de minimizar os sintomas, melhorar a resistência e diminuir o declínio no estado nutricional do paciente oncológico. 
Por fim, esse trabalho não visa esgotar a busca ou o estudo acerca deste assunto. Pela relevância do tema, faz-se necessário mais estudos a respeito da terapia nutricional em pacientes infanto-juvenis que passam pelo tratamento oncológico, já que há um grande declínio nos seus estados nutricionais. Uma limitação presente neste estudo, foi a aferição de alguns dados antropométricos (circunferência do braço e da cintura), impossibilitado devido a pesquisa ter sido feita de forma online.

\section{Referências:}

Araújo L. L., Barbosa J. M., Ribeiro A. P. G., Santos A. C. O. \& Pedrosa F. (2012). Nutritional status, dietary intake and serum levels of vitamin C upon diagnosis of cancer in children and adolescentes. Nutr Hosp. 27(2), 496-503.

Barreto, A. B. R., Haack, A., Santos, A. C. S. \& Silva, A. P. R. (2013) Perfil nutricional de pacientes pediátricos portadores de câncer, internados no Hospital da Criança de Brasília. Com. Ciências Saúde. 4(24), 315-20.

Caram, A. L. A. (2012). Desnutrição em Crianças até 12 Anos com Leucemia Atendidas no Grupo em Defesa de Criança com Câncer no Município de Jundiaí, SP. Revista Brasileira de Cancerologia. 2(58), 231-39.

Carraro, J. L., Schwartz R. \& Behling E. B. (2012). Comparação do estado nutricional obtido através de antropometria e bioimpedância em crianças e adolescentes submetidos a tratamento quimioterápico em um hospital do Sul do Brasil. Revista HCPA. 32(1), 35-41.

Carvalho, M., Albuquerque, Z. P., Monte, C. M. G., Aquino, K. K. N. C., Pinheiro, A. R. O. \& Gentil, P. C. (2005). Manual de atendimento da criança com desnutrição grave em nível hospitalar. 1. ed. Brasília. v. 1.

Feliciano, S. V. M., Santos, M. DE O. \& Pombo-de-Oliveira, M. S. (2019). Incidência e Mortalidade por Câncer entre Crianças e Adolescentes: uma Revisão Narrativa. Revista Brasileira de Cancerologia. 64, (3), 389-96.

Garófolo, A., Caran, E. M., Silva, N. S. \& Lopez, F. A. (2005). Malnutrition prevalence in children with solid tumors. Revista de Nutrição. 18(2), 193-200.

Garófolo, A. (2005). Guidance of nutritional support in critically ill children with cancer. Revista de Nutrição. 18(4), 513-27.

Garófolo, A. (2005). Diretrizes para terapia nutricional em crianças com câncer em situação crítica. Rev. Nutr. 18(4). Campinas, July-Aug.

Gomes, C. C. (2016) Estado nutricional e hormônios relacionados com o apetite em crianças e adolescentes com leucemia durante a fase de indução da quimioterapia. Dissertação de Mestrado, p. 65.

Instituto Nacional de Câncer. O que é câncer? 2020. <https://www.inca.gov.br/o-que-e-cancer>.

Lemos, P. S. M., Oliveira, F. L. C. \& Caram, E. M. M. (2014). Nutritional status of children and adolescents at diagnosis of hematological and solid malignances. Revista Brasileira de Hematologia e Hemoterapia. 36(6), 420-23.

Mutti, C. F., Cruz, V. G., Santos, L. F., Araújo, D., Cogo, S. B. \& Neves E. T. (2008). Perfil Clínico-epidemiológico de Crianças e Adolescentes com Câncer em um Serviço de Oncologia. Revista Brasileira de Cancerologia. 64(3), 293-300. <https://rbc.inca.gov.br/revista/index.php/revista/article/view/\%25a/4>.

Pereira, D. E. S., Andrade, E. S. A., Pedrosa, F. \& Barbosa, J. M. (2017). Estado Nutricional De Crianças E Adolescentes Em Início De Tratamento Oncológico Em Um Hospital De Referência Do Nordeste Do Brasil. VITTALLE - Revista de Ciências da Saúde. 29(1), 31-8.

Queiroz, C. M. M. A., Silva, M. A. G., Souza, A. P. B. \& Queiroz, D. J. M. (2018). Avaliação Do Perfil Nutricional De Crianças E Adolescentes com Câncer. Revista Saúde \& Ciência online. 7(2), 1-9.

Sgarbieri U. R., Fisberg M., Tone L.G. \& Latorre M. R. D. (2006). Nutritional assessment and serum zinc and copper concentration among children with acute lymphocytic leukemia: a longitudinal study. São Paulo Med J. 124(6), 316-20

Smiderle, C. A. \& Gallon, C. W. (2012). Desnutrição em oncologia: revisão de literatura. Revista brasileira de nutrição clínica. 27(4), 250-56.

Souza, J. A. \& Fortes, R. C. (2012). Qualidade de Vida de Pacientes Oncológicos: Um Estudo Baseado em Evidências. Revista de Divulgação Científica Sena Aires Julho-Dezembro. (22), 183-92.

Sousa, T. S., Santos, A. F., Santos, L. T. G., Sousa, P., Sousa, R. M. L., Martins, I. C. V. S., Dias, L. P. P., Maciel, M. G. \& Chein, M. B. C. (2016). Indicadores antropométricos em crianças com câncer em um hospital de referência. Rev Pesq Saúde. 17(3), 151-53.

SUÍÇA. World Health Organization. IMC para idade (5-19 anos). Genebra: WHO, 2007. <https://www.who.int/tools/growth-reference-data-for-5to19years/indicators/bmi-for-age\#> 